\title{
Control of wire melting behavior using coaxial hybrid solid wire*
}

\author{
by Nakamura Terumi **, Hiraoka Kazuo *** and Tanaka Manabu ***
}

\begin{abstract}
The wire melting behavior in pure Ar shielding gas can be controlled with a coaxial hybrid solid (CHS) wire which has a coaxial double structure with a different composition in its inner and outer parts. This wire can prevent the generation of a column of liquid metal (CLM) at the wire tip due to the difference in the materials properties of the inner and outer parts and stabilize MIG welding in pure Ar shielding gas (Ar-MIG welding). We examine the effects of the material properties (melting temperature, specific heat and thermal conductivity) on the wire melting behavior, then propose and show the effectiveness of a design guide for the CHS wire by carrying out a simulation and a welding examination on the new CHS wire which was developed based on this design guide.
\end{abstract}

Key Words: $\quad$ MIG arc welding, Pure Ar shielding gas, Column of liquid metal, Wire melting behavior, Coaxial hybrid solid wire, Instability, Simulation

\section{Introduction}

Coaxial hybrid solid (CHS) wires have been developed to carry out stable MIG welding in pure Ar shielding gas ${ }^{1,2)}$. The characteristics of the weld metal can be improved using the MIG welding in pure Ar shielding gas (Ar-MIG welding) since the amount of oxygen in weld metal, which deteriorates the characteristics of weld metal, decreases ${ }^{3)}$. However, this Ar-MIG welding is not used due to arc instability. If we use a conventional solid wire in pure Ar shielding gas, a column of liquid metal (CLM) is formed at the wire tip, and this CLM causes the Ar-MIG welding to become unstable. Therefore, it becomes essential to shorten the CLM to stabilize the Ar-MIG welding.

CHS wires have been developed to shorten this CLM ${ }^{1,2)}$. The composition of the inner and outer parts were selected so that the amount of melting in the inner part became greater than that of the outer part. Instead of generating a CLM, a big droplet was obtained; therefore, stable Ar-MIG welding was achieved.

The melting behavior depends on the material properties of the inner and outer materials, such as melting temperature, specific heat and thermal conductivity. This paper discusses the influence of the material properties on the wire melting behavior using a wire melting model of a CHS wire ${ }^{4)}$ first, then proposes a design guide for the development of a CHS wire.

\section{Wire melting simulation of CHS wire}

\subsection{Effect of material properties on wire melting behavior}

${ }^{*}$ Received: 2010.11 .12

${ }^{* *}$ Member, National Institute for Materials Science

${ }^{* * *}$ Member, Joining and Welding Research Institute Osaka University
Simulations were carried out to investigate the influence of the material properties on the wire melting behavior ${ }^{4)}$. Figure 1 (a) and (b) show the cross-section of the CHS wire and the CHS wire model, respectively. Steel was used for the outer part, and the inner part was varied based on the material properties. First, we investigated the effect of the melting temperature of the inner part of the wire on the wire melting behavior by changing the melting temperature of the inner part to $1273 \mathrm{~K}$ and $1981 \mathrm{~K}$.

Figure 2 shows the results of the simulation on the wire tip shape and temperature distribution. The simulated welding conditions were a welding current of $280 \mathrm{~A}$ and a wire feed rate of $170 \mathrm{~mm} / \mathrm{s}$. Fig. 2 (b) shows an image of the Inconel-Steel CHS wire (Fig. 1 (a)). In Fig. 2 (a), the melting temperature of the inner part of the wire was set to $1981 \mathrm{~K}$ which is higher than that of Inconel. The wire tip developed a convex shape, and the wire became extended. In Fig. 2 (c), the melting temperature of the inner part was set to $1273 \mathrm{~K}$ which is lower than that of Inconel. The wire tip developed a concave shape, and the wire became shorter than that of the Inconel-Steel CHS wire. Therefore, the use of low melting temperature materials in the inner part was found to be effective for shortening the CLM.

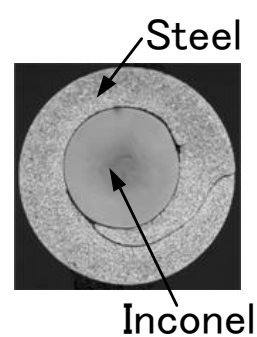

(a)

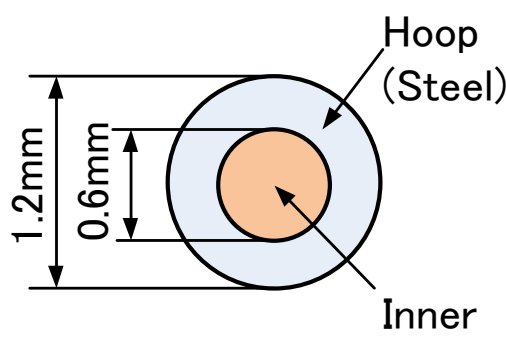

(b)

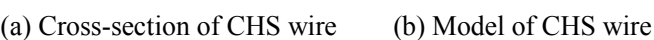
Fig. 1 Coaxial hybrid solid (CHS) wire 
Next, we showed the effect of the specific heat by changing the value of the specific heat of the inner part of the wire in Fig. 3. Inconel-Steel CHS wire is shown in Fig. 3 (b). In Fig. 3 (a), the specific heat of the inner part of the wire was set to twice that of Inconel. The wire tip developed a convex shape, and the wire became longer than that of the Inconel-Steel CHS wire. In Fig 3 (c), the specific heat of the inner part was set to half of that of Inconel. The wire tip shape became a trapezoid, and the wire became shorter than that of the Inconel-Steel CHS wire. The use of small specific heat materials in the inner part was also found to be effective for shortening the CLM.

Finally, we showed the effect of thermal conductivity. We changed the thermal conductivity of the inner part of the wire and presented the results in Fig. 4. The Inconel-Steel CHS wire is shown in Fig. 4 (b). The thermal conductivity of the inner part was set to twice and to half of that of Inconel in Fig. 4 (a) and Fig. 4 (c), respectively. The length of the wire is almost the same as that of the Inconel-Steel CHS wire under both conditions. The wire tip of Fig. 4 (c) and the isothermal line of $800 \mathrm{~K}$ are both sharp. The wire tip acquired a pencil-type shape, and the CLM formed quite easily. In contrast, both the tip shape of Fig. 4 (a) and the isothermal line of $800 \mathrm{~K}$ are round. To obtain the concave trapezoidal shape, a larger thermal conductivity is necessary. Therefore the effect of suppressing the generation of the CLM is small, compared to that of the melting temperature and the specific heat.

We also investigated the relationship between the length of the center part $L_{c e n t e r}$ and the material properties of the inner part. The length of the center part is defined in the illustration of Fig. 5.

Figure 6 shows the effects of the melting temperature of the inner part, the specific heat of the inner part and the thermal conductivity of the inner part. The horizontal axis is normalized by the values of the material properties of Inconel. The following ratios were used: $T_{\text {inner }} / T_{\text {Inconel }}, C_{\text {inner }} / C_{\text {Inconel }}, \lambda_{\text {inner }} / \lambda_{\text {Inonel }}$ where $T_{\text {inner }}$ is the melting temperature of the inner part, $T_{\text {Inconel }}$ is the melting temperature of Inconel, $C_{\text {inner }}$ is the specific heat of the inner part, $C_{\text {Inconel }}$ is the specific heat of Inconel, $\lambda_{\text {inner }}$ is the thermal conductivity of the inner part, and $\lambda_{\text {Inonel }}$ is the thermal conductivity of Inconel. The plot in Fig. 6 (a) has the largest slope indicating that the melting temperature has the largest influence on $L_{\text {center }}$. A slight change in the melting temperature causes a large change in $L_{c e n t e r}$. The length of $L_{\text {center }}$ and the ratio of $T_{\text {inner }} /$ $T_{\text {Inconel }}$ show a proportional relation within the range of computation. On the contrary, the effect of the specific heat was small compared to that of the melting temperature. A large change in $C_{\text {inner }} / C_{\text {Inconel }}$ is necessary if $L_{\text {center }}$ is to show the same change as that of Fig. 6 (a). The influence of the thermal conductivity on the $L_{c e n t e r}$ is the smallest among the material properties. The slope in Fig. 6 (c) is negative, and this tendency differs from that of the melting temperature and specific heat. Through these plots, we can clarify the effects of the material properties on the CHS wire melting behavior.

\section{Distance from contact tip $L_{C}(\mathrm{~mm})$}

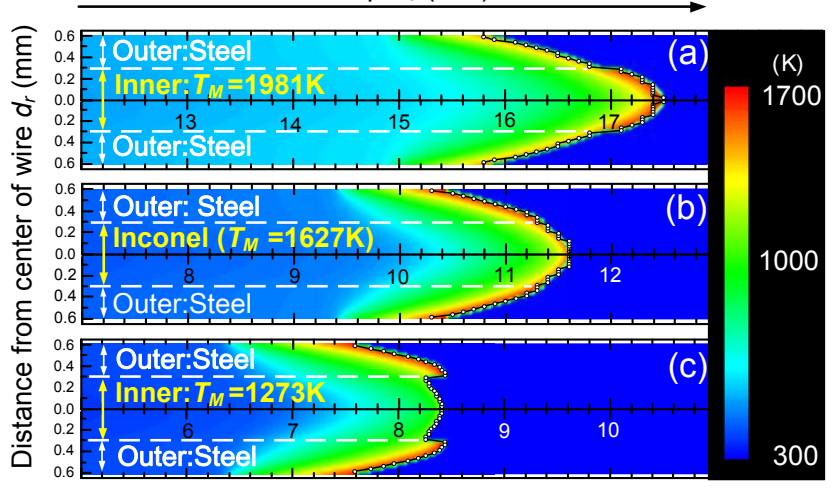

Fig. 2 Effect of the melting temperature of the inner part of the wire on the wire melting behavior $(I=280 \mathrm{~A})$

Distance from contact tip $L_{C}(\mathrm{~mm})$

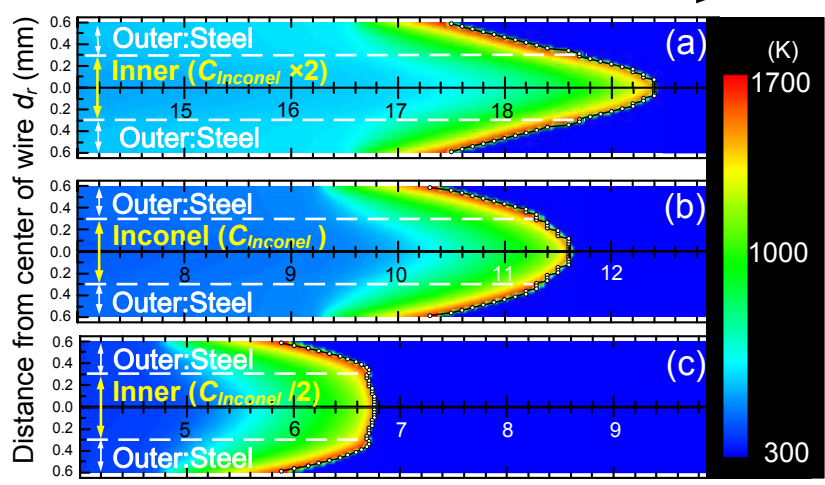

Fig. 3 Effect of the specific heat of the inner part of the wire on the wire melting behavior $(I=280 \mathrm{~A})$

Distance from contact tip $L_{C}(\mathrm{~mm})$

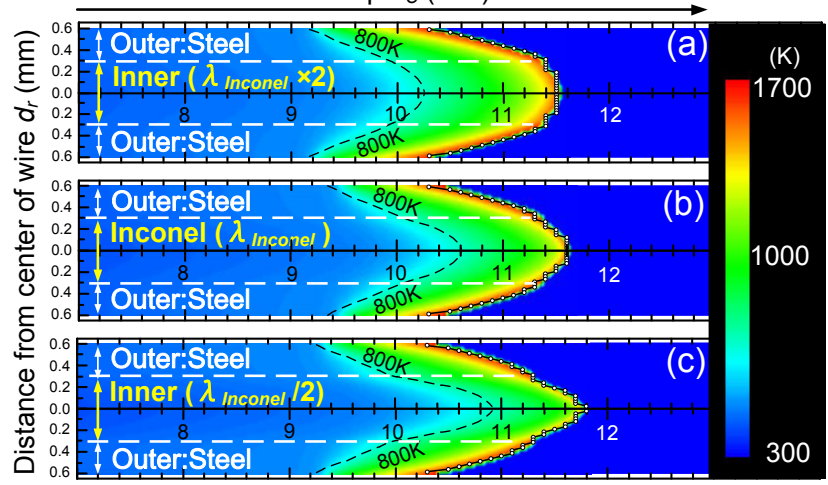

Fig. 4 Effect of the thermal conductivity of the inner part on the wire melting behavior $(I=280 \mathrm{~A})$ 


\section{Result and discussion}

\subsection{Design guide for $\mathrm{CHS}$ wire}

Appropriate materials must be selected for the inner and outer parts to shorten the CLM. Therefore, we have proposed a design guide for the CHS wire that shortens the CLM and enables steady Ar-MIG welding. The design guide for the selection of materials

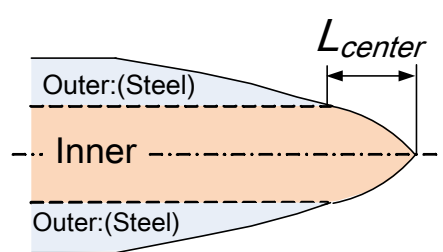

(a) $L_{\text {center }} \geqq 0$

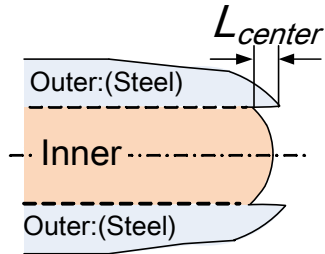

(b) $L_{\text {center }}<0$
Fig. 5 Definition of the length of center area

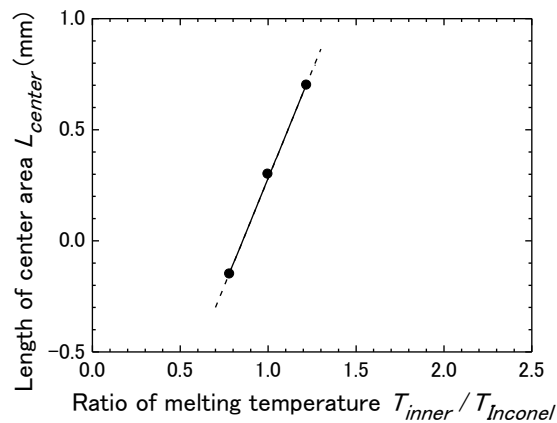

(a) Effect of melting temperature

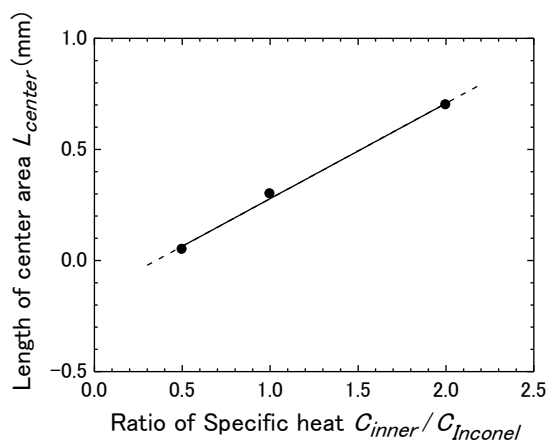

(b) Effect of specific heat

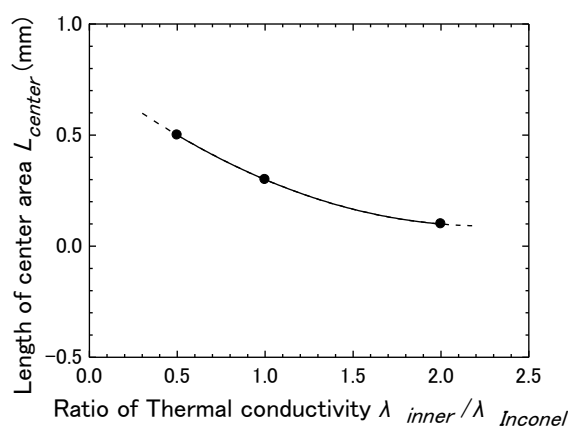

(c) Effect of thermal conductivity

Fig. 6 Effect of inner material properties on the length of center area is shown in Table 1. First, materials should be selected so that the melting temperature of the inner part is lower than that of the outer part. Next, the specific heat at the inner part should be smaller than that of the outer part. Finally, the thermal conductivity of the inner part should be larger than that of the outer part.

We designed a new CHS wire using this design guide. Three materials $(\mathrm{Cu}$, Steel and $\mathrm{Ti})$ were used. The melting temperature of $\mathrm{Cu}$, Steel and Ti are $1356 \mathrm{~K}, 1809 \mathrm{~K}$ and $1958 \mathrm{~K}$, respectively. The specific heat and the thermal conductivity at various temperatures are shown in Fig. 7. To shorten the CLM in pure Ar shielding gas, we selected $\mathrm{Cu}$ as the material for the inner part for the following reasons. (i) the melting temperature is low, (ii) the specific heat is small, (iii) the thermal conductivity is large. Ti or Steel is appropriate for the outer material. The melting temperatures of both materials are higher than that of $\mathrm{Cu}$, and thermal conductivities of both materials are almost the same level. However, the specific heat of steel is higher than that of $\mathrm{Ti}$; therefore, we selected steel as the material for the outer part.

Table 1 Design guide of CHS wire.

\begin{tabular}{lccc}
\hline & $\begin{array}{c}\text { Melting } \\
\text { temperature }\end{array}$ & Specific heat & $\begin{array}{c}\text { Thermal } \\
\text { conductivity }\end{array}$ \\
\hline Inner part & Low & Small & Large \\
\hline Outer part & High & Large & Small \\
\hline
\end{tabular}

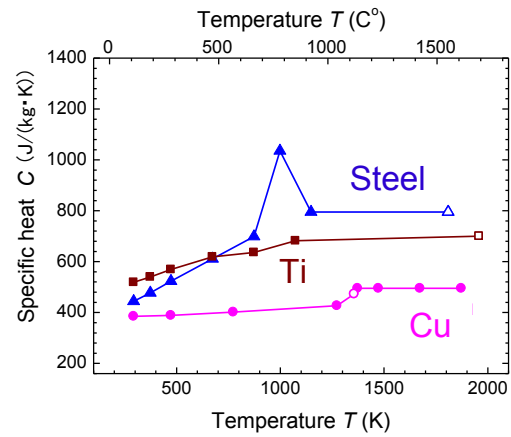

(a) Specific heat

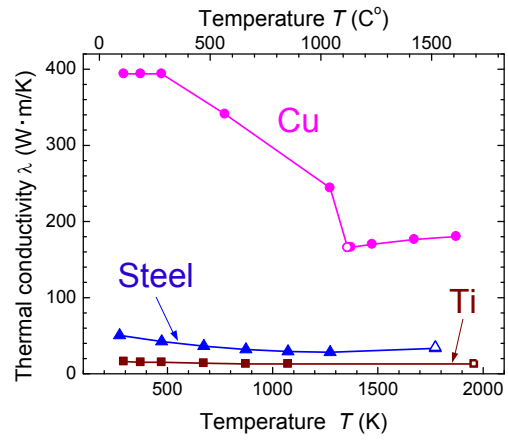

(b) Thermal conductivity

Fig. 7 Material properties of $\mathrm{Cu}$, Steel and $\mathrm{Ti}^{5}{ }^{5}$ 


\subsection{Welding behavior of $\mathrm{Cu}$-Steel CHS wire}

A simulation was carried out to investigate the wire melting behavior. The result presented in Fig. 8 shows that the wire tip has a concave shape. Thus, a long CLM was not generated in this $\mathrm{Cu}$-Steel CHS wire.

The welding behavior of the $\mathrm{Cu}$-Steel CHS wire is shown in Fig. 9 (a). The CLM did not form; therefore, stable welding was possible and good bead shape was obtained (Fig. 9 (c)). Figure 9 (b) shows the longitudinal sections after the welding. The amount of melting at the center part was larger than that of the outer part.

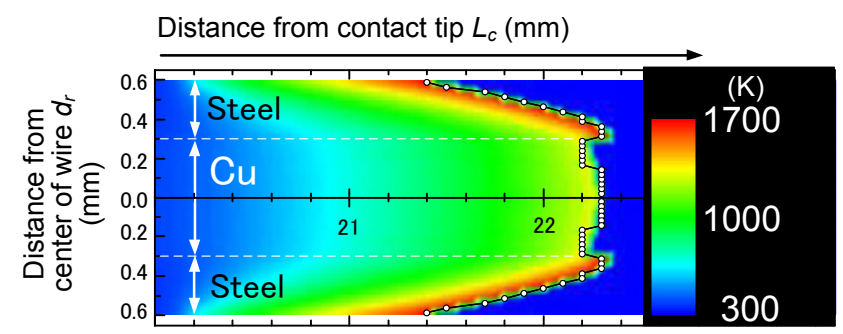

Fig. 8 Simulation of Cu-Steel CHS wire $(I=280 \mathrm{~A})$

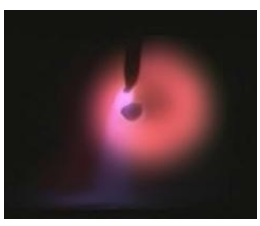

(a) Welding behavior

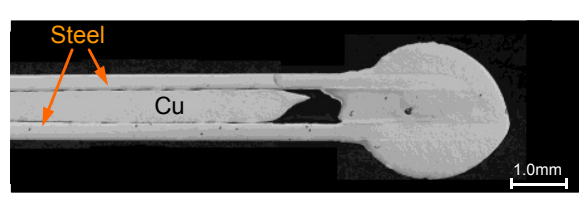

(b) Longitudinal section of wire

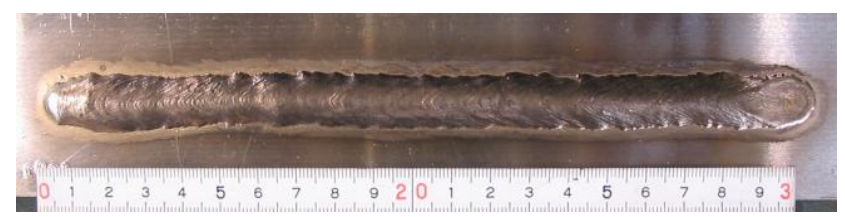

(c) Bead shape

Fig. 9 Welding result of $\mathrm{Cu}-\mathrm{Steel} \mathrm{CHS}$ wire $(I=280 \mathrm{~A})$

\section{Conclusions}

We investigated the effect of the material properties on the wire melting behavior to control the wire melting behavior in pure $\mathrm{Ar}$ shielding gas. The results are as follows.

The material properties of the inner and outer parts of the wire change the melting behavior. The influence of melting temperature is large, followed by specific heat and thermal conductivity.

We proposed a design guide for the CHS wire. We selected the materials so that the melting temperature of the inner part is lower than that of the outer part, the specific heat of the inner part is smaller than that of the outer part, and the thermal conductivity of the inner part is larger than that of the outer part.

$\mathrm{A} \mathrm{Cu}$-Steel CHS wire based on this design guide was produced. This developed wire did not generate any CLM in pure $\mathrm{Ar}$ shielding gas, and stable welding was possible. Therefore, we were able to show the effectiveness of the design guide for the CHS wire.

\section{Acknowledgements}

This research was supported by KAKENHI (18360358) and was achieved by collaboration with NIPPON WELDING ROD Co., Ltd., which is supported by the New Energy Industrial Technology Development Organization (NEDO), Japan.

\section{Reference}

1) T. Nakamura and K. Hiraoka: Development of stable MIG welding in pure Ar gas by New-Hybrid Wire, International Institute of Welding, Prague (2005), IIW Doc. 212-1080-05.

2) T. Nakamura, K. Hiraoka and S. Zenitani: Improvement of MIG welding stability in pure Ar shielding gas using a small amount of oxygen and a coaxial hybrid solid wire, Science and Technology of Welding and Joining, 13-1 (2008), 25-32.

3) S. Zenitani, T. Nakamura, K. Hiraoka and K. Shinozaki, Ar-MIG arc behavior in local addition of small amount of oxygen into shielding gas, Quarterly Journal of the Japan Welding Society, 25-1 (2007), 187-195. (in Japanese)

4) T. Nakamura and K. Hiraoka: Modeling of Wire melting behavior of coaxial hybrid solid wire in gas metal Arc welding in a pure Argon shielding gas, Welding in the World, 53- 7/8 (2009), R158-R165.

5) Metal Data book, The Japan Institute of Metals edited, Maruzen, 2004. 